\title{
EDITORIAL
}

\section{Propagation of Space Objects}

Martin Lara1, Rodolpho Vilhena de Moraes ${ }^{1,2}$, Diogo Merguizo Sanchez², Antonio Fernando Bertachini de Almeida Prado²

A ctual, important problems of space dynamics are related to the protection of the Earth and its close space environment. This includes the study of disposal strategies for artificial satellites, the real-time propagation of space catalogues of thousands of objects, the fast prediction of orbit evolution of fragmentation models, among others. Accurate predictions of orbit dynamics require the integration of complete force models, including both gravitational and non-gravitational effects. The most accurate integration is expected from numerical methods. However, in many cases, it is important to have available tools for the instant propagation of the orbit evolution, as opposite to precise ephemerides computation. These tools can be implemented with the help of modern perturbation methods, which, in addition to the mean element propagation, allow for the recovery of short-period effects to provide osculating elements.

Within a perturbative approach, the highest frequencies of the motion, which normally have small amplitudes, are analytically filtered via averaging procedures. From the mathematical point of view, the averaging is the result of a transformation from old to new variables, which, in reference to perturbed Keplerian motion, are called osculating and mean elements, respectively. This transformation is rarely attained in closed form, and one feels satisfied with computing the first terms of the Taylor series expansion of the transformation. The averaged equations depend only on long period angles and, therefore, are integrated numerically with very long step sizes. The short-period effects, if required, can be recovered at any step of the integration by the simple evaluation of the analytical expressions of the averaging transformation.

The averaging transformation can be performed either directly over the variation of parameters equations, using the generalized method of averaging (Bogoliuvov and Mitropolski, 1961), or in the Hamiltonian function, using canonical perturbation theory. For the latter, the Lie transforms method (Hori 1966, Deprit 1969) has the advantage - over Poincarés nouvelle method (Poincaré, 1893) or its von Zeipel-Brouwer variant (Ferraz-Mello, 2007) - of providing the canonical transformation of the averaging in explicit form, which makes the computation of higher orders in the perturbation approach simpler.

Analytical and semi-analytical theories based on averaging have been used in the propagation of catalogs of space objects since the beginning of the space era. Different algorithms based on Brouwer's theory (Brouwer, 1959) still serve for this task, and the generation of the so-called two-line elements is based on them (Hoot and Roehrich 1980; Vallado et al., 2006). More sophisticated theories were later implemented, as Draper semi-analytic satellite theory (DSST; McClain, 1977) or analytic orbit propagation program (AOPP; Coffey et al., 1995) in the United States, the numeric-analytic theory THEONA, developed at the Keldysh Institute of Applied Mathematics (Golikov, 1990), or the higher-order semi-analytical theory of the mean orbital motion, developed at the Observatoire de la Cote d'Azur (Métris and Exertier, 1995).

The main goal of the Sciences without borders project "Development and implementation of a semi-analytical theory for orbit propagation of artificial satellites based on Lie transforms", under the leadership of Rodolpho Vilhena de Moraes, Antonio F. Bertachini de Almeida Prado and Martin Lara, is the design and implementation of an efficient semi-analytical theory for the fast propagation of the osculating elements of space orbits in the close Earth environment. The theory will be based on the Lie transforms method, which is specifically designed for automatic computation by machine, and will explore the possibilities of polar-nodal variables, as well as of non-singular variables based on them, for improving the performance in the evaluation

1.Instituto de Ciência e Tecnologia - São José dos Campos/SP - Brazil 2.Instituto Nacional de Pesquisas Espaciais - São José dos Campos/SP - Brazil.

Author for correspondence: Antônio Fernando Bertachini de Almeida Prado - Instituto Nacional de Pesquisas Espaciais | Av. dos Astronautas, 1758 - Jardim da Granja | CEP: 12.227-010 - São José dos Campos/SP - Brazil | Email: antonio.prado@inpe.br 
of the very long Fourier series, which provide the short-period corrections, which can be made of tens of thousands of terms. The perturbation model should include the main perturbations, affecting Earth artificial satellites in the satellite regions of interest, namely the regions including low Earth orbits (LEO), geo-stationary orbits (GEO), mean Earth orbits (MEO) for traditional constellations of global navigation satellite systems, and high ellipticity orbits (HEO). Hence, the perturbation model must take into account: the effects of the J2-J8 zonal harmonics of the geopotential in closed form, as well as second order effects of $\mathrm{J} 2$ also in closed form; tesseral effects for the most common resonances (the 2:1 resonance affecting GPS orbits, the 5:3 resonance affecting Galileo disposal orbits, and the 1:1 resonance affecting the geosynchronous regime); lunisolar perturbations in the mass-point approximation, including at least the effects of the terms P2-P4 of the Legendre polynomials expansion of the thirdbody perturbation for the Moon, and the effect of the Legendre polynomial of second degree for the Sun; solar radiation pressure effects and drag. Future evolutions of the theory should make the semi-analytical propagation of uncertainties as well as the use of the theory for orbit determination possible.

The team members are experts in perturbation theory and have contributed to the understanding of third-body perturbations, resonances in the GPS regime and they also have developed new algorithms for the efficient computation of the Lie transforms theory. The development of the goals of the project is expected to provide new results of direct application to the fields of Aerospace Engineering, Mathematics, Celestial Mechanics, and Software Computing. In particular, the research topic will apply to actual problems of serious concern in space community, as is the case of Space Situational Awareness. Besides, it is expected that interested students may be challenged by the different research lines encompassed by the project. This interest would naturally materialize in Ph.D. thesis and Master's dissertation.

\section{REFERENCES}

Bogoliuvov, N.N. and Mitropolski, Y.A, 1961, "Asymptotic Methods in the Theory of Nonlinear Oscillations", Gordon and Breach, New York, USA.

Brouwer, D., 1959, "Solution of the Problem of Artificial Satellite Theory without Drag", Astronomical Journal, Vol. 64, No. 1274, pp. 378396.

Coffey, S.L., Neal, H.L., Segerman, A.M. and Travisano, J.J., 1995, "An Analytic Orbit Propagation Program for Satellite Catalog Maintenance", Advances in the Astronautical Sciences, Vol. 90, No. 2, pp. 1869-1892.

Deprit, A., 1969, "Canonical Transformations Depending on a Small Parameter", Celestial Mechanics, Vol. 1, No. 1, pp. 12-30. doi: 10.1007/BF01230629

Ferraz-Mello, S., 2007, "Canonical Perturbation Theories: Degenerate Systems and Resonance", Astrophysics and Space Science Library, Vol. 345, Springer, New York, USA, 341p.

Golikov, A.R., 1990, "Numeric-Analytical Theory of the Motion of Artificial Satellites of Celestial Bodies", Preprint No. 70 of Keldysh Institute of Applied Mathematics, Russian Academy of Sciences, Moscow, Russia.
Hori, G.I, 1966, "Theory of General Perturbation with Unspecified Canonical Variable", Publications of the Astronomical Society of Japan, Vol. 18, No. 4, pp. 287-296.

Hoot, F.R. and Roehrich, R.L., 1980, "Models for Propagation of the NORAD Element Sets", Project SPACETRACK, Rept. 3, U.S. Air Force Aerospace Defense Command, Colorado Springs, USA.

McClain, W.D., 1977, "A Recursively Formulated First-Order Semianalytic Artificial Satellite Theory Based on the Generalized Method of Averaging", Volume 1, Computer Sciences Corporation CSC/TR-77/6010. Silver Spring, MD, United States

Métris, G. and Exertier, P., 1995, "Semi-Analytical Theory of the Mean Orbital Motion", Astronomy and Astrophysics, Vol. 294, pp. 278-286.

Poincaré, H., 1893, "Les Méthodes Nouvelles de la Mécanique Céleste", Tome II, Gauthier-Villars et Fils, Paris, France.

Vallado, D.A., Crawford, P., Hujsak, R. and Kelso, T.S., 2006, "Revisiting Spacetrack Report \#3", Paper AIAA 2006-6753. 